Check for updates

Cite this: RSC Adv., 2018, 8, 30887

Received 16th July 2018

Accepted 27th August 2018

DOI: $10.1039 / \mathrm{c} 8 \mathrm{ra06034g}$

rsc.li/rsc-advances

\section{Novel oligopeptide nanoprobe for targeted cancer cell imaging $\dagger$}

\author{
Wen-Qiang Ding, Si-Yong Qin, (D) * Yin-Jia Cheng, Yi-Han Ma and Ai-Qing Zhang* \\ Here we designed and constructed a tryptophan-phenylalanine-phenylalanine-tryptophan (WFFW) \\ tetrapeptide, which generated photostable and tunable fluorescence emission signals from $340 \mathrm{~nm}$ to \\ $500 \mathrm{~nm}$. The WFFW tetrapeptide could self-assemble into a spherical nanostructure with enhanced \\ fluorescence intensity. Driven by $\pi-\pi$ stacking and hydrogen bond interaction, WFFW co-assembled \\ with arginine-glycine-aspartic acid (RGD) modified WFFW to form a cancer-targeted fluorescent \\ nanoprobe, which could selectively image the cancer cells.
}

\section{Introduction}

Effective cancer diagnosis in its early stages affords the greatest chance for prolonging survival, and even curing cancers. ${ }^{1,2}$ In the past few decades, constant efforts have been dedicated to developing novel techniques for the effective detection of cancers. Among them, fluorescence-guided diagnostics has received wide attention because of its non-invasive, real-time, high sensitivity, etc. aspects. ${ }^{3,4}$ Until now, various fluorescent systems basing on organic fluorescent dyes, ${ }^{5,6}$ quantum dots (QDs), ${ }^{7-10}$ and upconversion nanoparticles (UCNPs) ${ }^{11,12}$ have been rapidly developed for cancer diagnosis. Among them, most fluorescent probes could respond to the changes in cancer-related species (e.g., reactive oxygen species, ${ }^{13}$ enzymes,${ }^{14}$ and ions ${ }^{15}$ ) or unique tumorous microenvironments (e.g., slight acidity, ${ }^{16}$ and hypoxia $\left.{ }^{17,18}\right)$, which achieved off-on fluorescence imaging. For instance, Siegwart's group developed a PEGylated near-infrared 4,4'-difluoro-4-bora-3a,4a-diaza-s-indacene (BODIPY) probe, which activated the turn-on fluorescence signal in cancer cells due to the acidic $\mathrm{pH} .{ }^{19}$ In this design, PEG length adjustment could provide control over micrometastasis tracking and was thus capable of imaging tiny $(<1 \mathrm{~mm})$ micrometastases in different cancer tissues. To quantitatively visualize the protease activity and $\mathrm{pH}$ in the tumor microenvironment, a cancer-triggered fluorescent nanoprobe was constructed, in which $\mathrm{pH}$-sensitive dye was located at $\mathrm{Fe}_{3} \mathrm{O}_{4}$ nanoparticles via a peptide substrate of matrix metalloprotease9 (MMP-9) to establish a Förster resonance energy transfer (FRET) system. ${ }^{20}$ Using a near-infrared fluorescent dye of Cy5.5 as an internal reference, an MMP-dependent fluorescence

College of Chemistry and Materials Science, South-Central University for Nationalities, Wuhan 430074, P. R. China. E-mail: sy-qin@mail.scuec.edu.cn; aizhang@scuec.edu.cn

$\dagger$ Electronic supplementary information (ESI) available: Details of the characterizations of peptides and peptide-based nanoprobes such as MALDI-TOF-MS, fluorescent spectra, and FT-IR. See DOI: 10.1039/c8ra06034g signal could quantitatively reflect MMP-9 activity. The overexpressed MMP-9 and lower $\mathrm{pH}$ detected by the probe represented a powerful tool to study abnormal tumor signatures in vivo.

Despite the great advancements, the fluorescence-guided nano-diagnostics for cancer cells is still challengeable. For example, the synthesis process of constructing fluorescent nanosystems is generally complicated, partly because some fluorescent dyes are insoluble and need to be modified with functional hydrophilic groups. FRET-mediated fluorescent imaging is always associated with seeking suitable acceptor and donor pairs that were connected by cleavable bonds with certain lengths. ${ }^{21,22}$ The sensitivity of the cleavable bonds and linker length also affect the imaging capability. Specially, fluorescent organic molecules often suffer from high susceptibility to photobleaching. ${ }^{23}$ As for QDs, long-term cytotoxicity also remains a concern for in vivo fluorescent imaging. ${ }^{24}$ In recent years, biocompatible peptides have been proposed to modify organic fluorescent molecules, which can not only enhance the solubility, manipulate the optical properties by changing the peptide sequences ${ }^{25}$ but also achieve the targeted protein ${ }^{26}$ and cancer cell imaging. ${ }^{27,28}$ Nevertheless, organic fluorescent dyes are also indispensable for the fluorescent diagnosis. Zhen and co-workers developed a tryptophan-phenylalanine (Trp-Phe) dipeptide nanoparticle modified with an aptamer for targeted cancer cell imaging and real-time monitoring the drug release. ${ }^{29}$ In the absence of any organic fluorescence dyes, the selfassembled dipeptide nanoparticle could shift the intrinsic fluorescent signal of peptide from ultraviolet to $423 \mathrm{~nm}$ for bioimaging. However, extrinsic targeting group was involved. Pure peptide-based fluorescent probe with targeting function is highly pursued, especially with a longer emission wavelength.

In this work, we designed and prepared a peptide-based fluorescent nanosystem for targeted cancer cell imaging (Fig. 1). A tryptophan-phenylalanine-phenylalanine-tryptophan tetrapeptide (WFFW) that could generate the photostable and adjustable fluorescence signals from $340 \mathrm{~nm}$ to $500 \mathrm{~nm}$ was 


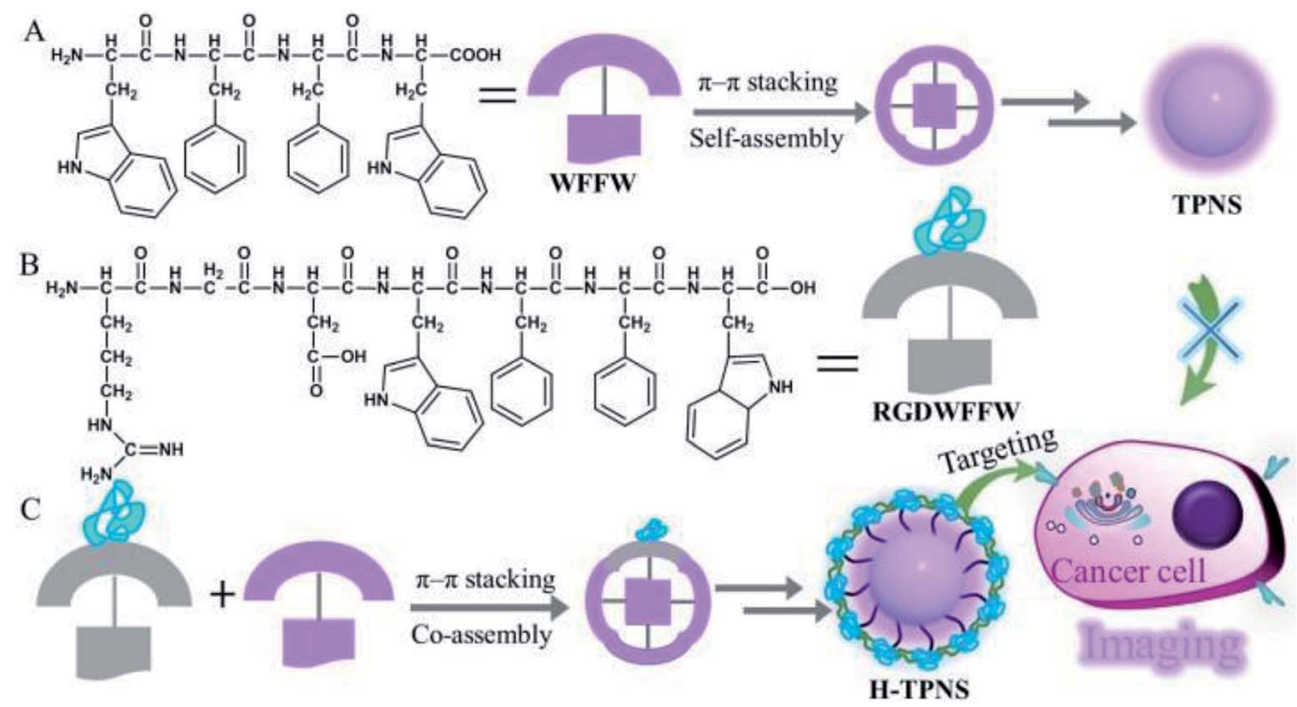

Fig. 1 (A) The chemical structure of WFFW tetrapeptide, which aggregated into spherical nanoprobes (TPNS) via molecular self-assembly. (B) The chemical structure of RGDWFFW heptapeptide. (C) Co-assembly of RGDWFFW and WFFW to construct the cancer-targeted nanoprobes (HTPNS) for cancer cell imaging.

fabricated (Fig. 1A). To endow the fluorescent probe with cancer-targeting, arginine-glycine-aspartic acid (RGD) modified WFFW (i.e. RGDWFFW) (Fig. 1B) was introduced to co-assemble with WFFW to form fluorescence enhanced nanoparticle for targeted cancer cell imaging (Fig. 1C).

\section{Experimental}

\subsection{Materials}

$N$-Fluorenyl-9-methoxycarbonyl (Fmoc) protected amino acids (Fmoc-Trp(Boc)-OH, Fmoc-Phe-OH, Fmoc-Arg(Pbf)-OH, Fmoc$\mathrm{Asp}(\mathrm{OtBu})-\mathrm{OH})$, Fmoc-Gly-OH, 2-chlorotrityl chloride resin (100-200 mesh, loading: $\left.0.97 \mathrm{mmol} \mathrm{g}{ }^{-1}, 1 \% \mathrm{DVB}\right), N$-hydroxybenzotriazole (HOBt) and $o$-benzotriazole- $N, N, N^{\prime}, N^{\prime}$-tetramethyluroniumhexafluorophosphate (HBTU) were purchased from GL Biochem. Ltd (Shanghai, China) and used as received. Trifluoroacetic acid (TFA) and diisopropylethylamine (DIEA) were provided by Aladdin Reagent Co. Ltd (Shanghai, China). Piperidine, $N, N$-dimethylformamide (DMF) and triisopropylsilane (TIS) were obtained from Sinopharm Chemical Reagent Co., Ltd (Shanghai, China) and used as received. Rhodamine B was obtained from Aladdin. Dulbecco's Modified Eagle's Medium (DMEM), fetal bovine serum (FBS), and 3-[4,5dimethylthiazol-2-yl]-2,5-diphenyltetra-zoliumbromide (MTT) were provided by Invitrogen Corp. All other chemical agents were purchased from Sinopharm Chemical Reagent Co., Ltd and used without any pre-treatment.

\subsection{Synthesis of peptide}

The peptides of WFFW and RGDWFFW were synthesized manually by solid phase method employing a standard Fmoc chemistry. ${ }^{30,31}$ After the attachment of amino acids to the resin, the peptide appended resin was washed with DMF, MeOH and DCM for three times, and then dried under vacuum overnight.
The dried resin was agitated with TFA (95\%), TIS $(2.5 \%)$ and $\mathrm{H}_{2} \mathrm{O}(2.5 \%)$ for $2 \mathrm{~h}$ at room temperature to detach the desired peptide from resin and remove the protective groups of aminoacid residues. The filtrate was collected and further condensed. The crude product was obtained by the precipitation with cold diethyl ether. After drying in a vacuum for $24 \mathrm{~h}$, the precipitate was dissolved in distilled water and freeze-dried. The molecular weight of the peptide was confirmed by matrix-assisted laser desorption/ionization time of flight mass spectrometry (MALDITOF-MS) (Fig. S1 in ESI†).

\subsection{Peptide self-assembly}

To prepare self-assembled tetrapeptide nanoprobes (TPNS), a certain amount of lyophilized WFFW powder was added into DMSO solution, yielding a peptide concentration of $50 \mathrm{mg}$ $\mathrm{mL}^{-1}$. Then the stock DMSO solution was diluted with phosphate-buffered saline (PBS, pH 7.4, $10 \mathrm{mM}$ ) to $0.5 \mathrm{mg}$ $\mathrm{mL}^{-1}$. The $\mathrm{pH}$ value of this system was adjusted to 11 with $\mathrm{NaOH}(1 \mathrm{M})$. After a few hours, The $\mathrm{pH}$ was reversely adjusted to 7.4 with $\mathrm{HCl}(1 \mathrm{M})$ to trigger the peptide self-assembly at room temperature. To construct the WFFW and RGDWFFW coassembled nanoprobes (H-TPNS), WFFW tetrapeptide and RGDWFFW heptapeptide (molar ratio $=1: 1$ ) were dissolved with DMSO, which was diluted with PBS to ensure the WFFW concentration of $0.5 \mathrm{mg} \mathrm{mL}^{-1}$.

\subsection{SEM characterization}

The morphology of self-assembled TPNS and H-TPNS was imaged by Scanning Electron Microscope (SEM) on a Scanning Electron Microscope Hitachi SU8010 (Japan) instrument with an accelerating voltage of $10 \mathrm{kV}$. Peptide solution with $0.5 \mathrm{mg}$ $\mathrm{mL}^{-1}$ was dropped onto a glass substrate and dried in air for $24 \mathrm{~h}$, which was further dehydrated under vacuum for the observation. 


\subsection{Nanoparticle size and $\zeta$-potential measurement}

The $\zeta$-potentials and average size of self-assembled nanoprobes were measured using a Nano-ZS ZEN3600 potentiometric particle size detector (Malvern Instruments) at $25{ }^{\circ} \mathrm{C}$. To investigate the size stability of nanoprobes, the average diameters of nanoprobes were monitored at different time intervals.

\subsection{FT-IR spectroscopy}

The FT-IR spectra of self-assembled TPNS and H-TPNS were obtained by Nexus 470 Spectrometer. The samples were freezedried and pressed into pellet with $\mathrm{KBr}$ powder before the measurement.

\subsection{Fluorescence spectroscopy}

Fluorescence emission spectra were measured on a Cary Eclipse Fluorescence Spectrophotometer (Hitachi F-7000) with light measured orthogonally to the excitation light.

\subsection{Photoluminescence quantum yield}

Photoluminescence quantum yields of TPNS and H-TPNS were obtained by comparison with a standard (rhodamine $\mathrm{B}$ in ethanol) and calculated according to the following formula: $\Phi_{\mathrm{X}}=\Phi_{\mathrm{ST}}\left(\operatorname{Grad}_{\mathrm{X}} / \operatorname{Grad}_{\mathrm{ST}}\right)\left(\eta_{\mathrm{X}}{ }^{2} / \eta_{\mathrm{ST}}{ }^{2}\right)$, where $\Phi$ is the fluorescence quantum yield, the subscripts ST and X denote standard and test respectively, Grad: the gradient from the plot of integrated fluorescence intensity vs. absorbance, $\eta$ : the refractive index of the solvent (water: 1.333, ethanol: 1.361). The data derived from the luminescence and the absorption spectra were shown in Fig. S2-S4. $\dagger$

\subsection{Cell culture}

Human cervix carcinoma (HeLa) cells and African green monkey kidney (COS7) cells were acquired from China Center for Typical Culture Collection (Wuhan, China), which were incubated in DMEM with $1 \%$ antibiotics (penicillin-streptomycin, $10000 \mathrm{U} \mathrm{mL}^{-1}$ ) and 10\% FBS in a fully humidified atmosphere of $5 \% \mathrm{CO}_{2}$ at $37^{\circ} \mathrm{C}$.

\subsection{In vitro cytotoxicity assay}

The cytotoxicity of the self-assembled peptide nanoprobes against HeLa and COS7 cells was examined by MTT assay. ${ }^{32,33}$ In detail, HeLa and COS7 cells were seeded with a density of $6 \times 10^{3}$ cells per well in a 96-well plate. The plate was then incubated for $24 \mathrm{~h}$ at $37^{\circ} \mathrm{C}$. For each group, $100 \mu \mathrm{L}$ of peptide solution with various concentrations was added and incubated with cells for $2 \mathrm{~h}$. Then, the medium containing peptide was removed and 20 $\mu \mathrm{L}$ of MTT solution ( $5 \mathrm{mg} \mathrm{mL} \mathrm{m}^{-1}$ ) was added to each well and incubated for $4 \mathrm{~h}$. Subsequently, the medium was removed and a certain of DMSO was added to dissolve formazan extraction. The absorbance of each hole at $570 \mathrm{~nm}$ was measured using a microplate reader (Bio-Rad, Model 550, USA). The relative cell viability of nanodrugs was calculated as: cell viability $(\%)=$ $\left(\mathrm{OD}_{570 \text { (sample) }} / \mathrm{OD}_{570 \text { (control) }}\right) \times 100$.

\subsection{Confocal laser scanning microscopy (CLSM)}

Laser confocal microscopy (Nikon C1-Si TE 2000, Japan) was used to observe the targeting ability of nanoprobe to different cells. First, the HeLa and COS7 cells were seeded into a 6-well plate with the cell density of $2 \times 10^{5}$ cells per well. After the culture in $37^{\circ} \mathrm{C}$ for $24 \mathrm{~h}$, the culture medium was discarded and the cells were washed with PBS for three times. Then, the TPNS and H-TPNS with concentration of $0.5 \mathrm{mg} \mathrm{mL}^{-1}$ were added to the HeLa and COS7 cells to culture for $30 \mathrm{~min}$, respectively. Finally, the residual medium was sucked out, and the cells were washed with PBS for three times. The fluorescence was observed with CLSM with a excitation wavelength of $405 \mathrm{~nm}$.

\section{Results and discussion}

To construct peptide-based fluorescent probe, two tetrapeptides with the sequences of WFFW and WFWF were proposed, since these peptides held the aromatic residues that can contribute to the self-assembly and fluorescent emission. ${ }^{29,34}$ First, the inherent fluorescent property of two tetrapeptides was investigated basing on fluorescent spectrometer in DMSO, where peptide molecules were expected to exist in less aggregated state. As shown in Fig. 2A, both of two tetrapeptides exhibited strong fluorescent signal around $475 \mathrm{~nm}$ under the excitation wavelength of $400 \mathrm{~nm}$. Both tetrapeptides exhibited similar fluorescent intensity under the same experimental conditions, showing the great potential of both tetrapeptides for potential fluorescence imaging. Thus, either of these could be selected as the fluorescent probe model and WFFW tetrapeptide was taken for the following study. To achieve the fluorescence-guided imaging, the optical property of WFFW tetrapeptide was then exploited in PBS. As shown in Fig. 2B, the maximum emission wavelength shifted to $500 \mathrm{~nm}$ at same excitation wavelength of $400 \mathrm{~nm}$. The concentration-dependent fluorescent intensity of WFFW tetrapeptide was also recorded. The fluorescence signal showed a descent trend once sequentially diluting the tetrapeptide solution with PBS. Interestingly, the fluorescence intensity showed a leaping between $0.10 \mathrm{mg} \mathrm{mL}^{-1}$ and $0.25 \mathrm{mg}$ $\mathrm{mL}^{-1}$. As we know, the fluorescence intensity is generally proportional to the sample concentration. And the abnormal emission leaping could be ascribed to the occurrence of selfassembly at the concentration range of $0.10-0.25 \mathrm{mg} \mathrm{mL}^{-1}$. Thus, all the investigation on WFFW tetrapeptide was carried
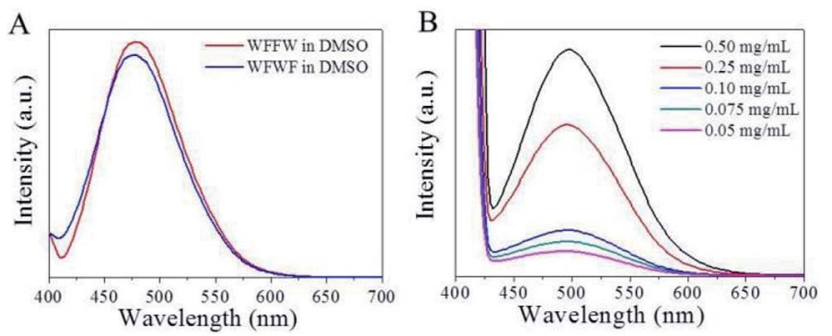

Fig. 2 (A) The fluorescence emission spectra of WFFW and WFWF in DMSO with $0.5 \mathrm{mg} \mathrm{mL}^{-1}\left(\lambda_{\text {ex }}=400 \mathrm{~nm}\right)$; (B) the fluorescence emission spectra of WFFW with different concentrations in PBS. 
out at a concentration of $0.5 \mathrm{mg} \mathrm{mL}^{-1}$, ensuring the occurrence of self-assembly. Interestingly, the unexpected fluorescence property that self-assembled nanoprobe exhibited enhanced fluorescent intensity than the monomeric state without selfassembly suggested the advantage of self-assembled nanoprobe over the molecular fluorescent peptide. Meanwhile, the photoluminescence quantum yield of TPNS was $11 \%$, which was similar with the self-assembled Trp-Phe nanoparticles. ${ }^{29}$

To investigate the morphology of self-assembled nanoprobe in PBS, SEM was employed. As shown in Fig. 3A, the WFFW probe exhibited a spherical structure, with a diameter of around $80 \mathrm{~nm}$. Meanwhile, the size of spherical peptide nanoprobe was measured by dynamic light scattering (DLS), which indicated an average diameter of $81 \mathrm{~nm}$ (Fig. 3B). The result was coincided well with the SEM observation, suggesting the successful fabrication of peptide nanoprobe. Notably, the biostability of self-assembled nanoparticles is a main concern for the biomedicine applications. Tracking the size as a function of time could provide some information about the stability. As shown in Fig. 3B, the average diameter of spherical nanoprobes was almost constant within $3 \mathrm{~d}$, which suggested the good size stability of TPNS. In addition, the photostability of fluorescent nanoprobes is also an important parameter for evaluating the imaging performance. Therefore, the time-dependent fluorescence intensity of self-assembled nanoprobe under natural light was recorded, accordingly. In the meanwhile, the organic fluorescent dye of rhodamine B was used as control. As shown in Fig. 3C, the fluorescence intensity of the peptide nanoprobe remained almost unchanged when exposed in natural light for 5 $\mathrm{d}$, whereas the fluorescence intensity of rhodamine B dropped quickly along with prolonging the time at room temperature (Fig. 3D). These results indicated the better photostability of

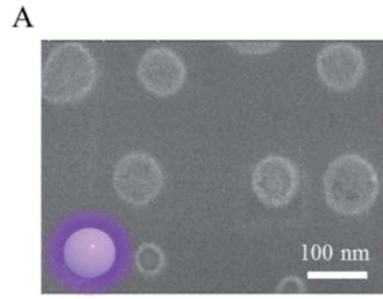

C

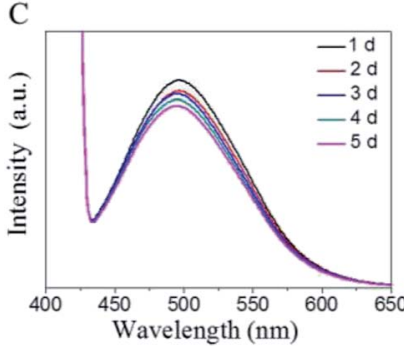

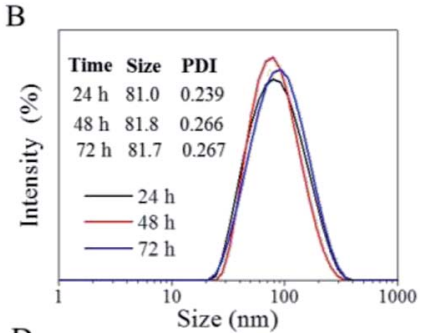

$\mathrm{D}$

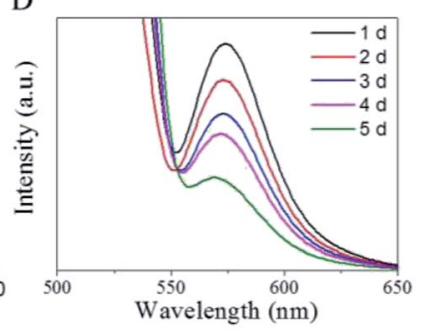

Fig. 3 (A) SEM image of the self-assembled TPNS. (B) The DLS profiles of spherical TPNS, indicating the average size of around $81 \mathrm{~nm}$. The stability investigation of TPNS was inserted. (C) The fluorescent emission spectra of WFFW nanoprobe within $5 \mathrm{~d}$, suggesting the good photostability. (D) The fluorescent emission spectra of organic dye rhodamine $\mathrm{B}$ as control. self-assembled peptide nanoprobe than that of the conventional dye of rhodamine $\mathrm{B}$.

It has been well documented that significant $\mathrm{pH}$ gradients exist within body and the tumor region is acidic. ${ }^{35}$ To probe the scope of self-assembled nanosphere for the cancer cell imaging, the fluorescent property of nanoprobe at different $\mathrm{pH}$ conditions was examined. Along with the elevation of $\mathrm{pHs}$, the intensity of maximum emission wavelength increased slightly, followed by a little decrease when the $\mathrm{pH}$ value exceeded 7.0 (Fig. S5, ESI $\dagger$ ). Meanwhile, a subtle red shift of fluorescent spectroscopy could be observed at different pHs, which might be ascribed to the enhanced $\pi-\pi$ stacking interaction among aromatic groups. ${ }^{34}$ Overall, the fluorescence emission of the nanoprobe was relatively stable in the $\mathrm{pH}$ range from 6.0 to 8.0, ensuring the validity of the self-assembled nanoprobe for cancer cell imaging. In addition, the emission wavelengths ranging from $360 \mathrm{~nm}$ to $500 \mathrm{~nm}$ were observed when tuning the excitation wavelength (Fig. S6, ESI $\dagger$ ), suggesting the adjustable fluorescence signal of the nanoprobe. To gain some insight into the molecular configuration of self-assembled nanoprobe, Fourier transform infrared (FT-IR) spectroscopy was used to determine the intermolecular arrangement within selfassembled nanoprobe (Fig. S7, ESI $\dagger$ ). The lyophilized TPNS showed a band at $\sim 1620 \mathrm{~cm}^{-1}$, suggesting the $\beta$-sheet like secondary conformation. ${ }^{36}$

As for the fluorescent nanoprobe for cancer cell detection, the selective imaging to targeted cells is critical. Therefore, RGD tripeptide, capable of targeting cancer cells that overexpressed the integrins, ${ }^{\mathbf{9}, \mathbf{1 0}, 37}$ was incorporated into WFFW tetrapeptide to construct the cancer-targeted fluorescent probe. Unfortunately, RGDWFFW exhibited weak fluorescence (Fig. 4A), suggesting that the introduction of RGD into WFFW peptide impaired the fluorescent property of TPNS. To achieve the targeted cancer cell imaging, an alternative strategy referring to the co-assembly of WFFW and RGDWFFW was proposed. Interestingly, the coassembly of WFFW and RGDWFFW did not affect the fluorescent emission of WFFW tetrapeptide. Instead, the fluorescent intensity increased greatly (Fig. 4A). The enhanced fluorescent signal indicated the occurrence of $\pi-\pi$ stacking interaction between WFFW and RGDWFFW.

The morphology of co-assembled nanoprobe was imaged by SEM. Similar with the nanosphere of self-assembled TPNS, the co-assembled H-TPNS also exhibited a spherical structure (Fig. 4B). Nevertheless, the size of co-assembled H-TPNS was around $150 \mathrm{~nm}$, which was bigger than the self-assembled TPNS. The reason for the different average diameters may be ascribed to the possibility that pure WFFW tetrapeptide possesses stronger intermolecular interaction than that of coassembled system consisting of WFFW and RGDWFFW, which yielded tight nanoparticle. DLS was also exploited to measure the size of co-assembled H-TPNS, showing an average diameter of around $150 \mathrm{~nm}$ that was consistent with the SEM result (Fig. 4C). The time-dependent size change also indicated the good size stability of H-TPNS. As for the concentrationdependent fluorescence emission of H-TPNS, a fluorescent jump between $0.25 \mathrm{mg} \mathrm{mL} \mathrm{m}^{-1}$ and $0.50 \mathrm{mg} \mathrm{mL}^{-1}$ could be observed (Fig. 4D), suggesting that the effective co-assembly 
A
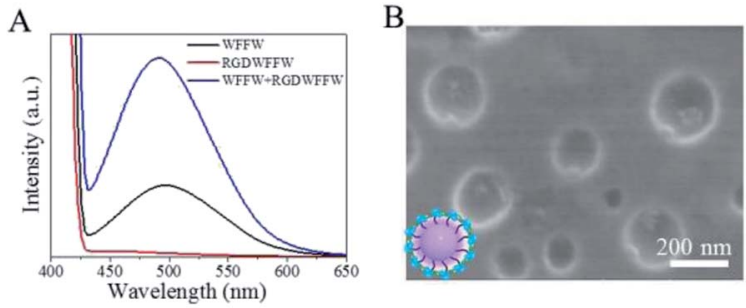

$\mathrm{C}$

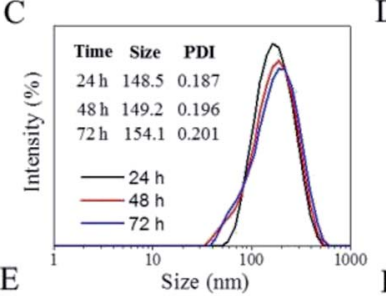

$\mathrm{D}$
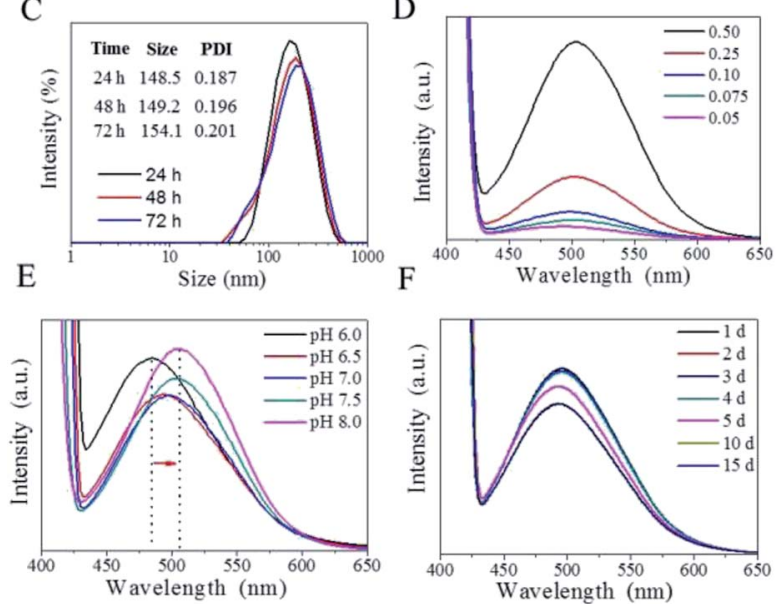

Fig. 4 (A) Fluorescent emission spectra of WFFW, RGDWFFW and WFFW + RGDWFFW. (B) SEM image of the co-assembled WFFW + RGDWFFW nanoprobes (H-TPNS). (C) The DLS profiles of spherical $\mathrm{H}$ TPNS within different time intervals. (D) The fluorescent emission spectra of WFFW + RGDWFFW with different concentrations (mg $\mathrm{mL}^{-1}$ ). (E and F) The fluorescent spectra of $\mathrm{H}$-TPNS at different $\mathrm{pHs}$ and time intervals.

concentration of WFFW and RGDWFFW was within the range. This peptide concentration for the co-assembly was a little higher than that of pure WFFW self-assembly, suggesting the stronger driving force for WFFW self-assembly. The selfassembled capacity reflected by peptide concentration discrepancy was agreement with the self-assembled potential obtained from the size discrepancy.

The fluorescent property of H-TPNS with different pHs and time intervals was also examined. Similar with TPNS, near $20 \mathrm{~nm}$ red shift of maximum fluorescent emission wavelength from $486 \mathrm{~nm}$ to $506 \mathrm{~nm}$ could be observed when changing the $\mathrm{pH}$ value from 6.0 to 8.0 (Fig. $4 \mathrm{E}$ ), suggesting the preferable alkalescence for the co-assembly of WFFW and RGDWFFW. The time-dependent fluorescent intensity also indicated the good photostability of H-TPNS (Fig. 4F). Also, the co-assembled probe exhibited tunable emission wavelengths ranging from $360 \mathrm{~nm}$ to $500 \mathrm{~nm}$ (Fig. S8, ESI $\dagger$ ), which broaden the application of fluorescent imaging. The similar FT-IR spectrum of coassembled WFFW and RGDWFFW nanosphere with pure selfassembled WFFW nanosphere indicated the similar secondary structures (Fig. S7 in ESI $\dagger$ ). In other words, the addition of targeted REDWFFW peptide did not affect the molecular arrangement pattern in self-assembly. In addition, the photoluminescence quantum yield of H-TPNS was $16 \%$, which was a little higher than the self-assembled TPNS nanoprobe.
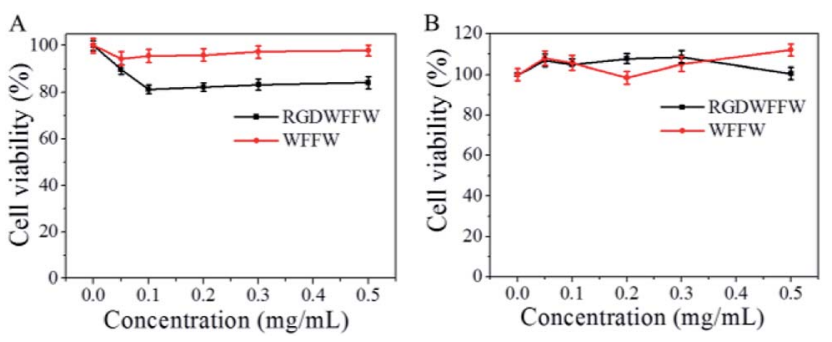

Fig. 5 Cell viability profiles of COS7 (A) and HeLa (B) cells after the incubation with WFFW and RGDWFFW for $2 \mathrm{~h}$, respectively.

Before the fluorescence-guided imaging for cancer cells, the biocompatibility of these nanoprobes was investigated against normal COS7 cells and cancerous HeLa cells. The MTT assay against COS7 cell line showed that both WFFW and RGDWFFW peptides exhibited little concentration-dependent cell viability in our observed concentration range (Fig. 5A). The cell viabilities of two nanoprobes in COS7 cells were higher than $80 \%$, suggesting the low cytotoxicity of these nanoprobes. The similar cell viability was also observed in the HeLa cell line (Fig. 5B), which further indicated the good biocompatibility of peptidebased fluorescent probe. Meanwhile, the $\zeta$-potentials of TPNS and H-TPNS nanoparticles were $-28.4 \mathrm{mV}$ and $-30.8 \mathrm{mV}$, accordingly (Fig. S9, ESI $\dagger$ ). The negative $\zeta$-potentials of nanoprobes suggested their potential stability in blood circulation due to weak adsorption with negative proteins in blood.

To confirm the suitability of nanoprobe for cancer cell targeting imaging, HeLa cell as a cancer cell model was investigated by CLSM. After the co-culture of co-assembled H-TPNS with HeLa cells for $30 \mathrm{~min}$, significant fluorescence was observed round HeLa cells (Fig. 6B). The strong fluorescence was attributed to the targeting recognition of RGD-bearing nanoprobe to the integrin overexpressed HeLa cells. To verify the specific recognition of H-TPNS to cancer cells, COS7 cells as a normal cell model that did not overexpress integrins was introduced as control. As shown in Fig. 6A, only very weak

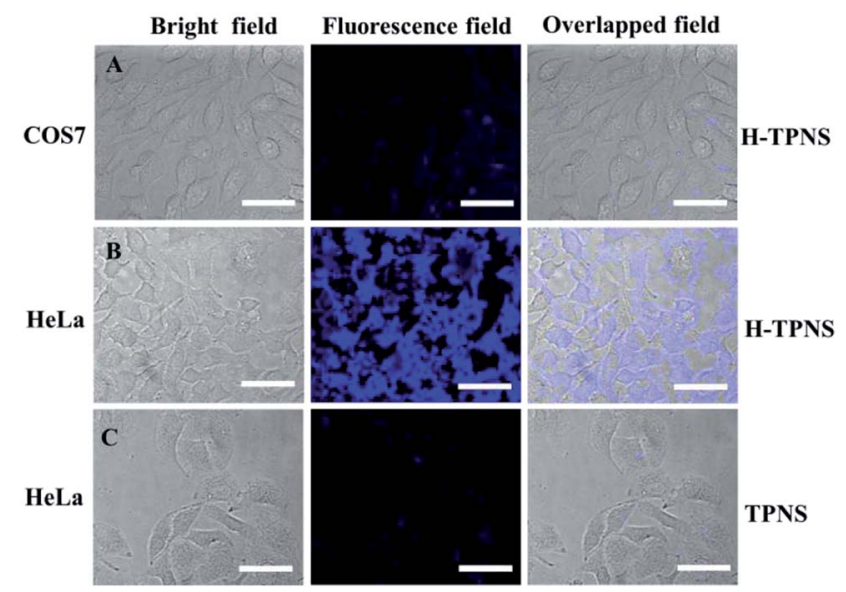

Fig. 6 Confocal images of fluorescent nanoprobe against HeLa and COS7 cells. (A) COS7 and (B) HeLa cells incubated with targeted nanoprobe of $\mathrm{H}$-TPNS at a concentration of $0.5 \mathrm{mg} \mathrm{mL}^{-1}$ for $30 \mathrm{~min}$. (C) HeLa cells incubated with nontargeted TPNS for same concentration and time. The scale bar is $36 \mu \mathrm{m}$. 
fluorescence was detected round COS7 cells at the same condition. Meanwhile, TPNS without RGD targeting group was also incubated with HeLa cells. As anticipated, little fluorescence signal was collected round HeLa cells (Fig. 6C), because of the lack of targeting group for the effective binding with cells. These results indicated the effective targeted cancer cell imaging of the co-assembled H-TPNS.

\section{Conclusions}

In summary, an aromatic WFFW tetrapeptide was synthesized, which self-assembled into spherical nanoparticle via $\pi-\pi$ stacking and hydrogen bond interaction. Without any organic fluorescent dyes, the self-assembled nanoparticle could generate the photostable and adjustable fluorescence signals from $340 \mathrm{~nm}$ to $500 \mathrm{~nm}$. To endow the fluorescent nanoparticle with targeting function to cancer cells, RGDWFFW was proposed, which co-assembled with WFFW to form fluorescence enhanced nanoprobe. This co-assembled nanoprobe successfully imaged the cancer cells, which gives insight into designing the pure peptide-based fluorescent probe for cancer diagnosis.

\section{Conflicts of interest}

There are no conflicts to declare.

\section{Acknowledgements}

We acknowledge the financial support from the National Natural Science Foundation of China $(51503227,51502352)$, the Natural Science Foundation of Hubei province of China (2017CFB528), the Fundamental Research Funds for the Central Universities, South-Central University for Nationalities (CZY18007), and Ministry-of-Education Key Laboratory for the Green Preparation and Application of Functional Materials, Hubei University.

\section{Notes and references}

1 A. M. Aravanis, M. Lee and R. D. Klausner, Cell, 2017, 168, 571-574.

2 K. J. McHugh, L. Jing, A. M. Behrens, S. Jayawardena, W. Tang, M. Gao, R. Langer and A. Jaklenec, Adv. Mater., 2018, 30, 1706356.

3 M. Gao, F. Yu, C. Lv, J. Choo and L. Chen, Chem. Soc. Rev., 2017, 46, 2237-2271.

4 D. Yue, M. Wang, F. Deng, W. Yin, H. Zhao, X. Zhao and Z. Xu, Chin. Chem. Lett., 2018, 29, 648-656.

5 G. Lin, P. N. Manghnani, D. Mao, C. Teh, Y. Li, Z. Zhao, B. Liu and B. Z. Tang, Adv. Funct. Mater., 2017, 27, 201701418.

6 H. Hu, Y. Zhang, S. Shukla, Y. Gu, X. Yu and N. F. Steinmetz, ACS Nano, 2017, 11, 9249-9258.

7 S. Jeong, J. Song, W. Lee, Y. M. Ryu, Y. Jung, S. Y. Kim, K. Kim, S. C. Hong, S. J. Myung and S. Kim, Nano Lett., 2017, 17, 1378-1386.
8 Z. Sun, Y. Zhao, Z. Li, H. Cui, Y. Zhou, W. Li, W. Tao, H. Zhang, H. Wang, P. K. Chu and X. F. Yu, Small, 2017, 13, 1602896.

9 L. Przysiecka, M. Michalska, G. Nowaczyk, B. Peplinska, T. Jesionowski, R. Schneider and S. Jurga, Colloids Surf., B, 2016, 146, 9-18.

10 B. R. Smith, Z. Cheng, A. De, J. Rosenberg and S. S. Gambhir, Small, 2010, 6, 2222-2229.

11 X. Ding, J. Liu, D. Liu, J. Li, F. Wang, L. Li, Y. Wang, S. Song and H. Zhang, Nano Res., 2017, 10, 3434-3446.

12 R. Tian, H. Zhang, H. Chen, G. Liu and Z. Wang, Adv. Sci., 2018, 5, 1800214.

13 H. Zhang, J. Liu, C. Liu, P. Yu, M. Sun, X. Yan, J. P. Guo and W. Guo, Biomaterials, 2017, 133, 60-69.

14 H. Cheng, S. Y. Li, H. R. Zheng, C. X. Li, B. R. Xie, K. W. Chen, B. Li and X. Z. Zhang, Anal. Chem., 2017, 89, 4349-4354.

15 D. P. Li, Z. Y. Wang, H. Su, J. Y. Miao and B. X. Zhao, Chem. Commun., 2017, 53, 577-580.

16 Y. Yue, F. Huo, S. Lee, C. Yin and J. Yoon, Analyst, 2017, 142, 30-41.

17 W. Piao, K. Hanaoka, T. Fujisawa, S. Takeuchi, T. Komatsu, T. Ueno, T. Terai, T. Tahara, T. Nagano and Y. Urano, J. Am. Chem. Soc., 2017, 139, 13713-13719.

18 J. N. Liu, W. Bu and J. Shi, Chem. Rev., 2017, 117, 6160-6224. 19 H. Xiong, H. Zuo, Y. Yan, G. Occhialini, K. Zhou, Y. Wan and D. J. Siegwart, Adv. Mater., 2017, 29, 1700131.

20 T. Ma, Y. Hou, J. Zeng, C. Liu, P. Zhang, L. Jing, D. Shangguan and M. Gao, J. Am. Chem. Soc., 2018, 140, 211-218.

21 Q. Su, W. Feng, D. Yang and F. Li, Acc. Chem. Res., 2017, 50, 32-40.

22 L. He, D. Q. Lu, H. Liang, S. Xie, C. Luo, M. Hu, L. Xu, X. Zhang and W. Tan, ACS Nano, 2017, 11, 4060-4066.

23 X. Wu and W. Zhu, Chem. Soc. Rev., 2015, 44, 4179-4184.

24 J. Fan, Y. Sun, S. Wang, Y. Li, X. Zeng, Z. Cao, P. Yang, P. Song, Z. Wang, Z. Xian, H. Gao, Q. Chen, D. Cui and D. Ju, Biomaterials, 2016, 78, 102-114.

25 S. Bai, S. Debnath, N. Javid, P. W. J. M. Frederix, S. Fleming, C. Pappas and R. V. Ulijn, Langmuir, 2014, 30, 7576-7584.

26 L. Feng, M. Chhabra, W. H. So, Q. Zhu, J. Xia and H. Sun, Chin. Chem. Lett., 2018, 29, 1147-1150.

27 X. Chen, P. S. Conti and R. A. Moats, Cancer Res., 2004, 64, 8009-8014.

28 X. Sun, Y. Li, T. Liu, Z. Li, X. Zhang and X. Chen, Adv. Drug Delivery Rev., 2017, 110-111, 38-51.

29 Z. Fan, L. Sun, Y. Huang, Y. Wang and M. Zhang, Nat. Nanotechnol., 2016, 11, 388-394.

30 C. M. Zhang, S. Y. Qin, Y. J. Cheng and A. Q. Zhang, RSC Adv., 2017, 7, 50425-50429.

31 S. Y. Qin, S. S. Xu, R. X. Zhuo and X. Z. Zhang, Langmuir, 2012, 28, 2083-2090.

32 S. Y. Qin, M. Y. Peng, L. Rong, H. Z. Jia, S. Chen, S. X. Cheng, J. Feng and X. Z. Zhang, Nanoscale, 2015, 7, 14786-14793.

33 S. Y. Qin, Y. J. Cheng, Z. W. Jiang, Y. H. Ma and A. Q. Zhang, Colloids Surf., B, 2018, 165, 345-354.

34 S. Qin, Y. Pei, X. Liu, R. Zhuo and X. Zhang, J. Mater. Chem. B, 2013, 1, 668-675. 
35 M. Karimi, A. Ghasemi, P. Sahandi Zangabad, R. Rahighi, S. M. Moosavi Basri, H. Mirshekari, M. Amiri, Z. Shafaei Pishabad, A. Aslani, M. Bozorgomid, D. Ghosh, A. Beyzavi, A. Vaseghi, A. R. Aref, L. Haghani, S. Bahrami and M. R. Hamblin, Chem. Soc. Rev., 2016, 45, 1457-1501.
36 K. Katayama, Y. Furutani, M. Iwaki, T. Fukuda, H. Imai and H. Kandori, Phys. Chem. Chem. Phys., 2018, 20, 3381-3387.

37 M. Peng, S. Qin, H. Jia, D. Zheng, L. Rong and X. Zhang, Nano Res., 2016, 9, 663-673. 